\title{
Miniature Nernstian oxygen sensor for deposition and growth environments
}

\author{
E. van Setten \\ MESA ${ }^{+}$Research Institute and Department of Applied Physics, University of Twente, \\ 7500 AE Enschede, The Netherlands \\ T. M. Gür \\ Geballe Laboratory for Advanced Materials, Stanford University, Stanford, California 94305 \\ D. H. A. Blank \\ MESA ${ }^{+}$Research Institute and Department of Applied Physics, University of Twente, \\ 7500 AE Enschede, The Netherlands \\ J. C. Bravman \\ Department of Materials Science and Engineering, Stanford University, Stanford, California 94305 \\ M. R. Beasley \\ Department of Applied Physics and the Geballe Laboratory for Advanced Materials, Stanford University, \\ Stanford, California 94305
}

(Received 25 October 2000; accepted for publication 15 October 2001)

\begin{abstract}
A miniature Nernstian-type oxygen sensor employing an ionically conducting stabilized zirconia solid electrolyte and an embedded internal reference electrode is developed and is tested in the temperature range $275-425^{\circ} \mathrm{C}$. It is intended for in situ monitoring of oxygen content in deposition and growth environments. Advantages of this sensor are in situ generation of the $\mathrm{Ni} / \mathrm{NiO}$ two phase internal reference thin film electrode using a solid-state oxygen Coulometric titration technique, its small size $(5 \mathrm{~mm} \times 10 \mathrm{~mm})$, flat geometry $(<1 \mathrm{~mm}$ thick), relatively low operating temperature, and the fact that the entire sensor can be immersed in the gaseous environment with the unknown oxygen content. The latter feature is distinctly different from commercially available Nernstian oxygen sensors, which employ two-compartment geometries. Depending upon temperature, the time constants for the sensor response to changes in oxygen pressure ranged from seconds to minutes, which is exceptionally fast for these moderately low temperatures. The small thermal mass and size of this single-compartment electrochemical sensor make it suitable for in situ and noninvasive real time monitoring of oxygen content during thin-film deposition or bulk growth of complex oxides. (C) 2002 American Institute of Physics. [DOI: 10.1063/1.1424906]
\end{abstract}

\section{INTRODUCTION}

Advanced materials often constitute the vital core of new technologies. For faster, cheaper, and more effective ways of developing new materials, it is imperative to have in situ control of primary synthesis parameters such as composition and temperature at the growing surface. Unfortunately, most methods employed for this purpose are necessarily empirical in nature and there is no easy and cost effective solution to this urgent problem. Secondary synthesis parameters and postmortem characterization of the resulting material are often the only tools available for fine tuning of the deposition or growth process. Naturally, development of new sensors and in situ analytical tools ${ }^{1,2}$ will greatly benefit these otherwise iterative ex situ methods that necessarily lead to expensive and slow progress.

Multicomponent oxides exhibit a wide range of scientifically interesting and technologically important properties. But their inherently complex phase relations and crystal structures pose difficult challenges for the synthesis of the desired phase with the correct oxygen stoichiometry. There is an intimate relationship between the stability of the oxide phase and the nature of the oxygen environment during deposition and growth. ${ }^{3,4}$ Similarly, their electronic transport properties often depend strongly upon, and may vary significantly with, oxygen nonstoichiometry. ${ }^{5}$ It is often the case, however, that control of oxygen content during oxide synthesis is accomplished indirectly by flow mixing or postmortem characterization of the resulting material. Unfortunately, these ex situ techniques are largely ineffective for rapid development of complex oxides. Hence, in situ monitoring and control of the local oxygen content around the growing oxide is important in order to obtain the desired phase in bulk or thin-film form with a prescribed and precise oxygen stoichiometry.

The purpose of this communication is to report the preliminary progress regarding the development of a miniature Nernstian-type oxygen sensor for the monitoring of oxygen in deposition and growth environments at moderate temperatures. As distinct from the commercially available large, tubular, and bulky oxygen sensors of this kind, this sensor is a single-compartment, flat device with an embedded reference electrode. It has a logarithmic response and is exceptionally sensitive to detect small quantities of oxygen in a gas mixture. The wide range of oxygen partial pressures it can accurately sense is limited only by the onset of electronic conduction in the ionically conducting solid electrolyte under 
extremely reducing conditions. In the case of stabilized zirconia, for example, the lower boundary of its electrolytic domain where the ionic and electronic transference numbers become 0.5 each (i.e., $50 \%$ of the electrical charge through the solid electrolyte is transported by ionic defects and the remaining $50 \%$ is transported by electronic species) is about $10^{-30} \mathrm{~atm}$ of oxygen partial pressure of $1000^{\circ} \mathrm{C}$, and $10^{-39}$ atm at $800{ }^{\circ} \mathrm{C} .{ }^{6}$ Consequently, the range of oxygen partial pressure where the zirconia solid electrolyte remains predominantly ionically conducting is extremely wide, covering oxygen activities from 5 atm down to $10^{-25}$ atm at temperatures below $1000{ }^{\circ} \mathrm{C}$. ${ }^{6}$ It is for this reason that this type of sensor, in principle, has the ability to detect and monitor extremely low oxygen partial pressures. The sensors in this study, however, have not been tested below $10^{-4}$ atm of oxygen partial pressure.

In contrast to the commercial oxygen sensors, which are bulky with internal gas cavities and poor thermal shock resistance, this single-compartment sensor possesses a low thermal mass due to its small size and is also nonobstructive in the deposition and growth environments. Naturally, these advantages also make this sensor of practical interest for in situ control and monitoring of oxygen content in other applications such as flue gases and mixtures, molten metals and alloys, steel-making, metal foundries, combustion processes, medical applications and devices, air management systems, electronic circuits, and hermetically sealed chips.

Currently, two types of high-temperature electrochemical oxygen sensors are commercially available. Both use stabilized zirconia as the oxide ion conducting solid electrolyte and operate at elevated temperatures $800-1000^{\circ} \mathrm{C}$. The first kind is a limiting current-type sensor ${ }^{7,8}$ which operates on the principle of diffusion limited current induced by the viscous flow of molecular oxygen through a small circular aperture from the measuring environment into a ceramic cavity that employs a stabilized zirconia solid electrolyte. This sensor has a linear response with respect to oxygen content but suffers considerably from interference effects from other oxygen containing chemical constituents in the environment. It also performs poorly at low oxygen concentrations where limited current plateaus are not well separated to offer satisfactory resolution for accurate determination of oxygen content. Hence, this type of sensor may not be well suited for deposition and growth environments with low oxygen content.

The second kind, which is of particular interest to this paper, is a logarithmic response concentration cell-type sensor operating on the Nernstian principle., ${ }^{9,10}$ Presently, all commercial Nernstian-type oxygen sensors have a twocompartment tubular geometry where the inside compartment is usually exposed to air that serves as the reference electrode for fixed and known oxygen activity, while the outside electrode senses the unknown oxygen content in the environment. This type of sensor - also known as $\lambda$ sensor — is widely used in the exhaust gas manifold of automobiles manufactured in Western countries. The accuracy of these commercial sensors is well documented down to $10^{-15} \mathrm{~atm}$ of oxygen partial pressures. ${ }^{11}$ These two-compartment sensors are bulky and prone to thermal and mechanical failure.
Their high operating temperatures require the use of bulky heaters. Maintaining gas-tight structural separation between the oxygen reference electrode compartment and the environment of interest is also a problem. These considerations make them unsuitable for monitoring the oxygen content in controlled environments for thin-film deposition and growth.

\section{MATERIALS ASPECTS}

The critical component of the Nernstian sensor is an oxide ion conducting solid electrolyte, such as stabilized zirconia, that allows selective and fast diffusion of oxide ions at elevated temperatures. Zirconia $\left(\mathrm{ZrO}_{2}\right)$ itself is nonconducting both electronically and ionically but displays considerable solid solubility (up to $20 \mathrm{~mol} \%$ ) for many alkali and rare-earth oxides at elevated temperatures. When the hightemperature cubic fluorite phase of zirconia is stabilized by doping with alkali or rare-earth oxides, the dopant cations substitute for the $\mathrm{Zr}^{+4}$ sites in the crystal structure giving rise to the creation of oxygen vacancies in order to maintain the charge neutrality of the crystal. Since the solubilities are high, the resulting oxygen vacancy concentrations in the doped material are also very high. For example, $10 \mathrm{~mol} \%$ $\mathrm{CaO}$ stabilized zirconia contains about $10 \mathrm{~mol} \%$ oxygen vacancy since each $\mathrm{Ca}^{+2}$ sitting in a $\mathrm{Zr}^{+4}$ site leads to the creation of one oxygen vacancy for charge compensation. By the same token, $8 \mathrm{~mol} \% \mathrm{Y}_{2} \mathrm{O}_{3}$ stabilized zirconia YSZ contains about $4 \mathrm{~mol} \%$ oxygen vacancies.

Such a high vacancy concentration, approaching a fraction of the Avogadro number, facilitates the selective diffusion of oxygen through this family of doped zirconia via a vacancy diffusion mechanism, giving rise to a high ionic conductivity. ${ }^{12}$ Moreover, the electrical conductivity remains predominantly ionic in nature even at elevated temperatures with practically no concomitant electronic conduction over a wide range of oxygen activities. In other words, the transference number for oxygen ions is near unity (i.e., $t_{I}>0.99$ ), indicating that almost all current through the solid electrolyte is carried by oxide ions.

\section{OPERATING PRINCIPLE}

Electrochemical oxygen sensing involves the electrode reaction for oxygen incorporation into YSZ whose elementary steps ${ }^{13}$ can collectively be summarized as

$$
\begin{aligned}
& \frac{1}{2} \mathrm{O}_{2(\mathrm{~g})}=\mathrm{O}_{\mathrm{ad}(\mathrm{M})}, \\
& \mathrm{O}_{\mathrm{ad}(\mathrm{M})}+V_{\mathrm{O}(\mathrm{YSZ})}^{00}+2 e_{(\mathrm{M})}^{\prime}=\mathrm{O}_{\mathrm{O}(\mathrm{YSZ})}^{\mathrm{X}},
\end{aligned}
$$

where, in accordance with Kröger-Vink notation, $V_{\mathrm{O}}^{00}$ indicates an oxygen vacancy in YSZ with an effective charge of +2 with respect to the perfect lattice, $\mathrm{O}_{\mathrm{O}}^{\mathrm{X}}$ is neutral oxygen occupying a normal oxygen lattice site in $\mathrm{YSZ}_{2} \mathrm{O}_{2(\mathrm{~g})}$ is molecular oxygen in the gas phase, $\mathrm{O}_{\mathrm{ad}(\mathrm{M})}$ is adsorbed oxygen on the metal electrode $(\mathrm{M})$, and $e_{\mathrm{M}}^{\prime}$ is an electron with an effective charge of -1 located in the metal electrode. Equation (1) represents the dissociative adsorption of molecular oxygen on the metal electrode surface. The adsorbed oxygen 

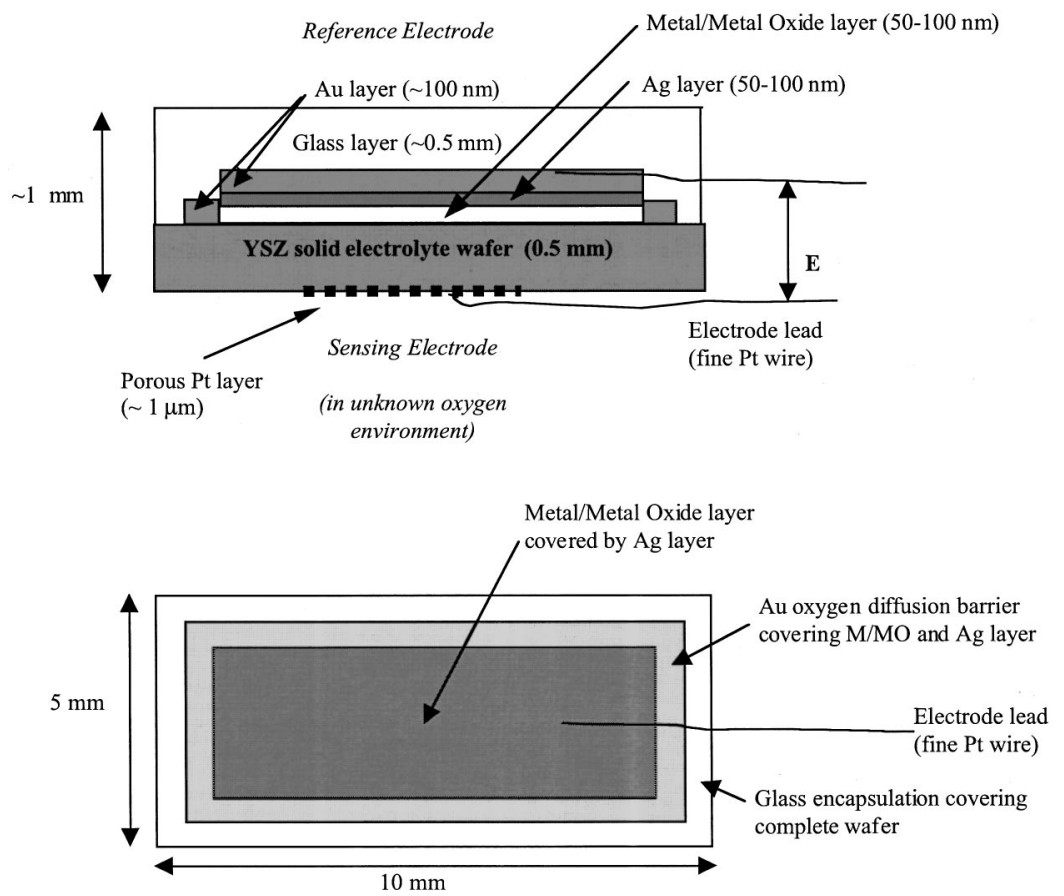

FIG. 1. Cross-section and top view of the schematic structure, dimensions, and individual thickness of the thin-film layers of the solid-state Nernstian oxygen sensor with embedded $\mathrm{Ni} / \mathrm{NiO}$ internal reference electrode. picks up two electrons from the metal electrode, surface diffuses to a charge transfer site at a three-phase boundary, and is incorporated into the vacancy site in YSZ as a neutral species in Eq. (2).

If there is a difference in the oxygen chemical potential across the solid oxide electrolyte, the resulting difference in the oxygen activities, $a_{\mathrm{O}_{2}}$, is related to the open circuit potential $E$ across the electrolyte by the Nernst equation:

$$
E=-R T / n F \ln \left(a_{\mathrm{O}_{2}}^{\prime} / a_{\mathrm{O}_{2}}^{\prime \prime}\right),
$$

where $R$ is the universal gas constant, $T$ denotes the temperature, $n$ is the gram equivalent for oxygen, and the superscripts indicate different oxygen activities on each side of the electrolyte. Assuming Reaction (1) is in equilibrium, i.e., dissociative adsorption of molecular oxygen on the metal electrode surface is sufficiently fast, and that ideal gas behavior is valid for these moderately elevated temperatures, Eq. (3) can be expressed as

$$
E=-R T / 4 F \ln \left(P_{\mathrm{O}_{2}} / P_{\mathrm{ref}} \mathrm{O}_{2}\right),
$$

where $P_{\text {ref }} \mathrm{O}_{2}$ is the reference oxygen pressure on one side of the electrolyte and $P_{\mathrm{O}_{2}}$ denotes the unknown oxygen pressure of the environment on the other side. If the oxygen activity on one side of the electrolyte is fixed by a suitable reference such as air or a metal/metal oxide two-phase mixture, then the unknown oxygen pressure $P_{\mathrm{O}_{2}}$ can readily be extracted from Eq. (4) which, in fact, represents the basic principle on which Nernstian-type oxygen sensors operate.

\section{MINIATURE SENSOR DESIGN AND FABRICATION}

The schematic design of the sensor is shown in Fig. 1. Single crystal wafers of $10 \mathrm{~mol} \% \quad \mathrm{Y}_{2} \mathrm{O}_{3}$ stabilized $\mathrm{ZrO}_{2}$ (YSZ) cut into $10 \mathrm{~mm} \times 5 \mathrm{~mm} \times 0.5 \mathrm{~mm}$ elements are used as the solid oxide electrolyte. Opposite flat surfaces of the YSZ are polished to different surface roughness using $20 \mu \mathrm{m}$ alumina on one side and $1 \mu \mathrm{m}$ diamond paste on the other. A thin layer of porous Pt deposited from an unfluxed Pt paste formulation (No. 6926 Engelhard Corp.) on the rough side of YSZ for better adhesion serves as the oxygen sensing electrode where Reactions (1) and (2) take place. The sensing electrode is completely exposed to the ambient environment whose oxygen content is to be determined and monitored. The Pt paste coating is fired in air at $900{ }^{\circ} \mathrm{C}$ for $1 \mathrm{~h}$ to remove the organic constituents and form a porous adhered layer less than $1 \mu \mathrm{m}$ thick. In order to make the electrical connection to this electrode, a 0.003-in. diam $(0.0762 \mathrm{~mm})$ fine Pt wire is embedded in a blob of Pt paste placed on top of the porous electrode layer and fired at $900{ }^{\circ} \mathrm{C}$ for $30 \mathrm{~min}$. Alternatively, Ag paste (SPI Supplies, Silver Paste "Plus" 30 GM) was used to attach the Pt wire to the electrode layer when lower temperatures (i.e., $250^{\circ} \mathrm{C}$ for $30 \mathrm{~min}$ ) were required for firing.

A key feature of this sensor design is the internal oxygen reference electrode formed electrochemically in situ and made of a suitable metal-metal oxide mixture that has sufficient electronic conductivity. The Gibbs phase rule dictates that the two phase metal-metal oxide binary equilibrium fixes the oxygen activity at a given temperature. Hence, this fixed and known oxygen activity serves as the oxygen reference potential against which the unknown oxygen content is obtained in accordance with Eq. (4). Another key feature is the encapsulation and physical isolation of the internal reference electrode from its gaseous environment so that it does not exchange oxygen with its surrounding. This is a critical design consideration for reliable performance of the sensor.

The next step in the process is the fabrication of the embedded, thin-film reference electrode on the smooth surface of the YSZ electrolyte. Two techniques, namely, direct current (dc) sputtering and pulsed laser deposition (PLD), have been used for depositing thin films of the precursor layer for the internal reference electrode. Although there is 
no fundamental reason not to start with the metal layer as the precursor, for various experimental reasons, we have found that starting out with the metal oxide precursor layer in preparation for the metal-metal oxide binary mixture gave more satisfactory results. Most important, this strategy eliminates inadvertent oxidation of the metal precursor layer during subsequent coating of the Au barrier layer, as well as the molar volume expansion during oxidation that may cause cracking of the encapsulation. If such cracks develop, oxygen from the ambient may leak into the embedded reference electrode, destroying the two-phase equilibrium giving rise to catastrophic failure of the sensor.

The initial experiments using $\mathrm{Cu}$ or $\mathrm{CuO}$ precursor layers to prepare the $\mathrm{Cu} / \mathrm{Cu}_{2} \mathrm{O}$ or $\mathrm{Cu}_{2} \mathrm{O} / \mathrm{CuO}$ reference electrodes were not successful partly due to high diffusivity and solubility of $\mathrm{Cu}$ in the $\mathrm{Au}$ and glass encapsulant layers in the case of $\mathrm{Cu} / \mathrm{Cu}_{2} \mathrm{O}$ and to insufficient electrical conductivity of the $\mathrm{Cu}_{2} \mathrm{O} / \mathrm{CuO}$ mixture. Thus the results reported in this article involve the use of $\mathrm{Ni} / \mathrm{NiO}$ reference electrodes only.

YSZ wafers that already have the Pt sensing electrodes on the rough face were coated with 50-100-nm-thick $\mathrm{NiO}$ films on the highly polished face. The $\mathrm{NiO}$ layers covering an area of $3 \mathrm{~mm} \times 6 \mathrm{~mm}$ were often deposited by PLD using a $248 \mathrm{~nm}$ excimer laser source and a Ni metal target. Oxygen partial pressure in the deposition chamber was $20 \mathrm{~Pa}$ while the single crystal YSZ wafers were maintained at room temperature. A nonporous 0.1-1- $\mu \mathrm{m}$-thick layer of Au deposited by PLD, sputtering, or from a paste formulation is used as the oxygen diffusion barrier that completely encapsulates the $\mathrm{NiO}$ layer. Typically, this is done by coating the $\mathrm{NiO}$ film by a $100 \mathrm{~nm} \mathrm{Au}$ layer using PLD at room temperature and a pressure of $2 \times 10^{-4} \mathrm{~Pa}$, which led to the best results. It should be noted, however, that attention must be given to thermodynamic considerations such as solid solubility and chemical compatibility between different layers in Fig. 1. For example, the solid solubility of $\mathrm{Ni}$ in $\mathrm{Au}$ is $13 \mathrm{~atm} \%$ at $500{ }^{\circ} \mathrm{C}$. This necessitates the insertion of a chemical barrier layer to prevent $\mathrm{Au}$ alloying with $\mathrm{Ni}$ in the $\mathrm{Ni} / \mathrm{NiO}$ reference electrode and affecting the local oxygen activity. Silver is found to be an effective barrier interlayer and provided the best results. The 50-100-nm-thick Ag interlayer was also deposited using PLD following the deposition of the $\mathrm{NiO}$ layer while keeping the YSZ wafer in the vacuum chamber. To ensure a good electrical connection with the reference electrode a fine $\mathrm{Pt}$ wire embedded in a $\mathrm{Au}$ paste formulation (Hanovia UR No. 01-FM) is placed on the Au layer and is fired in air at $850{ }^{\circ} \mathrm{C}$ to remove the organic binder. The last step of the encapsulation process involved the use of a Corning No. 7570 grade glass frit (softening point: $447^{\circ} \mathrm{C}$ ) to coat the $\mathrm{Au}$ barrier layer and achieve complete isolation of the internal reference electrode. The $\mathrm{PbO}$-based glass frit coating was fired at $500{ }^{\circ} \mathrm{C}$ for $30 \mathrm{~min}$. The thickness of the glass coating was approximately $0.5 \mathrm{~mm}$.

After encapsulation, the final stage of the fabrication process involved the generation of the $\mathrm{Ni} / \mathrm{NiO}$ internal reference electrode in situ, by partly decomposing the $\mathrm{NiO}$ embedded layer. Solid-state oxygen Coulometric titration ${ }^{14}$ was employed for this purpose. It involves the application of a small dc bias galvanostatically or potentiostatically across the YSZ solid electrolyte that serves as an oxygen pump selectively transporting small but precise amounts of oxygen. This corresponds to changing intermittently the composition of the NiO layer at each titration step by removing electrochemically controlled amounts of oxygen from the NiO/YSZ interface and subsequently allowing the cell the relax to its new equilibrium. The $\mathrm{Ni} / \mathrm{NiO}$ binary mixture forms when the oxygen activity at the interface eventually attains the thermodynamic value where $\mathrm{Ni}$ and $\mathrm{NiO}$ coexist in equilibrium at that temperature. The major advantage of using the Coulometric titration technique is the ability to generate the twophase $\mathrm{Ni} / \mathrm{NiO}$ internal reference electrode in situ.

\section{RESULTS AND DISCUSSION}

Of the 16 sensors that have been fabricated, only few were operational. The majority failed for mechanical, thermal, or chemical reasons at various stages of fabrication and testing. The sensors that were electrically and mechanically in tact were tested in air for the temperature dependence of their open circuit potentials using a temperature programmable heater. At a ramping rate of $5 \mathrm{~K} / \mathrm{min}$, the temperature coefficients for the open circuit potential of the sensors varied in the range from -0.46 to $-0.50 \mathrm{mV} / \mathrm{K}$, in good agreement with the expected value of $-0.488 \mathrm{mV} / \mathrm{K}$ for the $\mathrm{Ni} / \mathrm{NiO}$ equilibrium.

The sensors were tested for their response to changes in oxygen pressure in the temperature range $275-425^{\circ} \mathrm{C}$. The upper limit of the temperature range is set by the softening point of $447^{\circ} \mathrm{C}$ for the glass frit used for encapsulation. A 1 1/4-in.-diam and 18-in.-long quartz tube equipped with an 8-in.-long external cylindrical resistance heater is used for this purpose. In actual deposition chamber environment, heating of this miniature sensor to the desired operating temperature may be achieved using a thin Mo sheet heater. The sensor assembly can be placed close to the substrate to monitor oxygen activity during deposition of complex oxides films, but care should be taken to properly shield the sensing Pt electrode from the plasma during physical vapor deposition.

The sensor element is placed inside the quartz tube that is connected by a switching valve to a cylinder of $\mathrm{He}$ and a cylinder of air, so that the gases could be switched rapidly providing sudden changes in the oxygen environment of the sensor element. Even at these rather moderate temperatures of $275-425^{\circ} \mathrm{C}$, the sensors responded rapidly to changes in the oxygen activity indicating good sensitivity and sufficiently fast kinetics on the Pt sensing electrode. The response behavior of one of the sensors at $425^{\circ} \mathrm{C}$ is depicted in Fig. 2, which shows a full cycle switching from air to He gas and then back to air environment. The drop in open circuit potential upon switching to He flow corresponds to an oxygen partial pressure of $4.2 \times 10^{-4}$ atm which is typical for the oxygen impurity content in regular purity He gas cylinders.

It should be noted that the sensor response is of the order of seconds, impressively fast for these moderate temperatures. Based on these findings, this sensor may be operated at around $400-425^{\circ} \mathrm{C}$ for best results. And although it has only been tested down to about $10^{-4}$ atm of oxygen pressure, this 


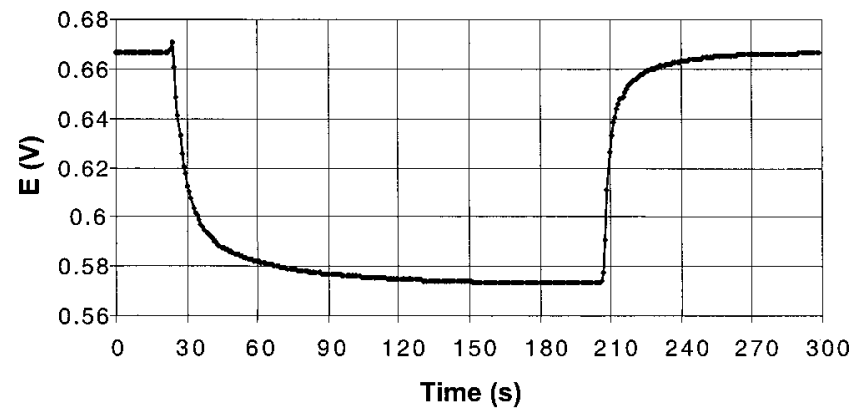

FIG. 2. Time-dependent variation of the open circuit potential of the sensor depicting the response at $425{ }^{\circ} \mathrm{C}$ to changes in the oxygen content as the environment around the sensor is switched from air to He gas and back to air.

sensor should be able to detect much lower oxygen pressures as does its commercial counterpart. This is because the zirconia electrolyte, which is primarily responsible for the accuracy and resolution of detection, remains predominantly ionically conducting down to extremely low oxygen partial pressures at these temperatures. ${ }^{6}$

The small, sudden increase of open circuit potential upon switching from air in the quartz jacket to flowing He by opening the He cylinder valve can be contributed to the cooling effect of the flowing gas. Indeed, the $3.8 \mathrm{mV}$ increase in the open circuit potential in Fig. 2 corresponds to a temperature drop of about $7{ }^{\circ} \mathrm{C}$, which agrees with experimental observations. It is also clear from Fig. 2 that there is asymmetry in the response behavior of the sensor going from high to low oxygen activity (i.e., from air to $\mathrm{He}$ ) as opposed to going from low to high activity (i.e., from He to air).

The sensor response to changes in the oxygen environment is adequately represented by an exponential function. Figure 3 shows a good fit to the experimental data for the early part of the response for $t<2 \mathrm{~min}$. Time constants derived from the best fit to the exponential decay in the sensor response are presented in an Arrhenius plot in Fig. 4. The scatter in the data is considerable and may be related to temperature variations resulting from the cooling effect caused by differences in gas flow rates as $\mathrm{He}$ and air are switched back and forth. The asymmetry in the sensor response to

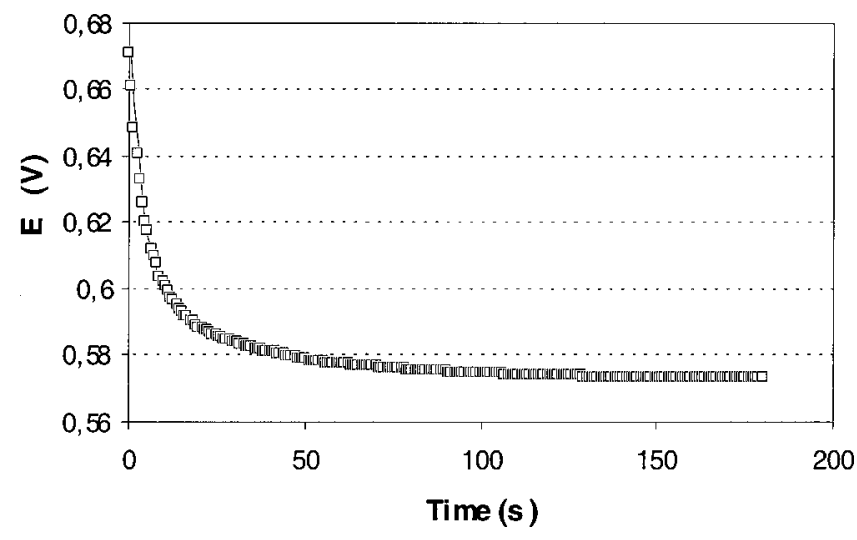

FIG. 3. Exponential fit of the simulated sensor response to the experimentally observed behavior of the sensor at $425^{\circ} \mathrm{C}$. The solid line represents the simulated response while the squares denote the experimentally measured data.

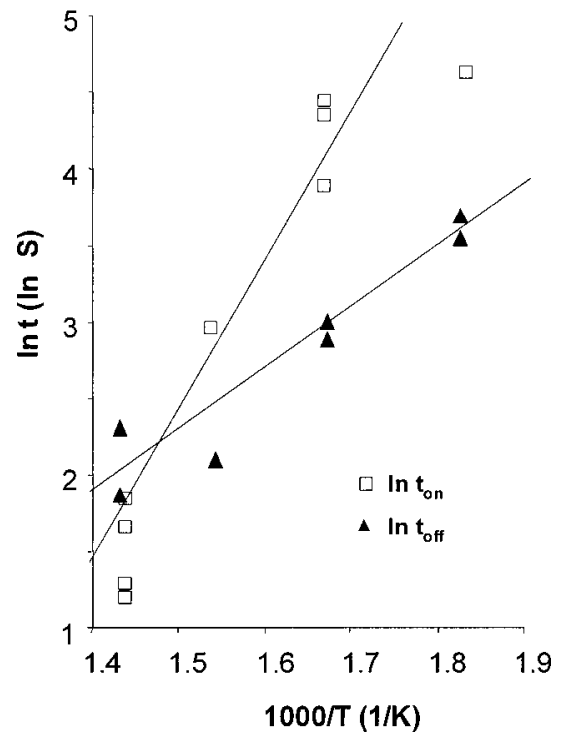

FIG. 4. Arrhenius plot for the time constants of sensors. The squares denote sudden decrease in oxygen activity switching from air to He, while filled triangles represent increase in oxygen activity from $\mathrm{He}$ to air.

changes in oxygen environment manifests itself as distinct activation energies. Clearly, the activation energy is much larger for the case of decreasing oxygen activity $(78 \mathrm{~kJ} / \mathrm{mol})$ in the environment (i.e., from air to $\mathrm{He}$ ) than it is for the case where the oxygen activity is increasing $(38 \mathrm{~kJ} / \mathrm{mol})$. Unfortunately, it is not possible at this time to attribute these energies to a particular physical phenomenon.

It is well known, however, that molecular oxygen readily adsorbs on Pt surfaces with very low activation energy of about $12 \mathrm{~kJ} / \mathrm{mol}^{15}{ }^{15}$ Below $120 \mathrm{~K}$, oxygen adsorbs predominantly in molecular form and dissociates into atomic oxygen when Pt is heated to $300 \mathrm{~K},{ }^{16}$ with an activation energy of 33 $\mathrm{kJ} / \mathrm{mol}$ for the dissociation of molecular adsorbed oxygen. ${ }^{17}$ Conversely, the values of the activation energies for oxygen desorption from Pt surfaces are much higher and vary up to $213 \mathrm{~kJ} / \mathrm{mol}^{17,18}$ depending upon the particle size, site, and crystal surface of Pt as well as the extent of atomic oxygen coverage on the Pt surface. Obviously, these literature values do not reconcile with the activation energies extracted from Fig. 4. However, the literature data clearly point to the highly activated nature of the desorption rate compared to adsorption of oxygen on $\mathrm{Pt}$ surfaces. Indeed, temperature programmed desorption (TPD) studies of oxygen on Pt surfaces with varying degrees of oxygen exposure history consistently indicate that desorption is observed only at temperatures above $600-800 \mathrm{~K}^{15,18}$

This qualitative argument suggests that Reaction (1) may be governing the overall sensor response behavior and this is consistent with the surface kinetics of the $\mathrm{Pt} / \mathrm{O}_{2}$ system. ${ }^{15-18}$ The dissociative adsorption of molecular oxygen on $\mathrm{Pt}$, which is in effect what happens when the oxygen activity in the environment is increased, seems to be much faster (i.e., smaller time constants) than the process of associative desorption of atomic oxygen from the Pt surface when the oxygen activity is decreased. The latter of course has a longer time constant, as observed here. 


\section{ACKNOWLEDGMENTS}

This work was supported by the NSF-MRSEC Program through the Center for Materials Research (CMR) at Stanford University and benefited from the facilities and equipment made available by the NSF-MRSEC Program through CMR.

${ }^{1}$ N. J. C. Ingle, R. H. Hammond, M. R. Beasley, and D. H. A. Blank, Appl. Phys. Lett. 75, 4162 (1999).

${ }^{2}$ M. A. Kelly, M. L. Shek, P. Pianetta, T. M. Gür, and M. R. Beasley, J. Vac. Sci. Technol. A 19, 2127 (2001).

${ }^{3}$ J. Li, T. M. Gür, R. Sinclair, S. S. Rosenblum, and H. Hayashi, J. Mater. Res. 9, 1499 (1994).

${ }^{4}$ B. T. Ahn, V. Y. Lee, R. Beyers, T. M. Gür, and R. A. Huggins, Physica C 167, 529 (1990).

${ }^{5}$ R. Beyers, B. T. Ahn, G. Gorman, V. Y. Lee, S. S. P. Parkin, M. L. Ramirez, K. P. Roche, J. E. Vazquez, T. M. Gür, and R. A. Huggins, Nature (London) 340, 619 (1989).
${ }^{6}$ J. W. Patterson, E. C. Bogren, and R. A. Rapp, J. Electrochem. Soc. 114, 752 (1967).

${ }^{7}$ K. Saji, J. Electrochem. Soc. 134, 2430 (1987).

${ }^{8}$ B. Y. Liaw and W. Weppner, J. Electrochem. Soc. 138, 2478 (1991).

${ }^{9}$ K. Saji, H. Kondo, T. Takeuchi, and I. Igarashi, J. Electrochem. Soc. 135, 1686 (1988).

${ }^{10}$ N. Hara and D. D. Macdonald, J. Electrochem. Soc. 144, 4158 (1997).

${ }^{11}$ J. E. Federer, J. Electrochem. Soc. 131, 755 (1984).

${ }^{12}$ T. M. Gür, I. D. Raistrick, and R. A. Huggins, Mater. Sci. Eng. 46, 53 (1980).

${ }^{13}$ T. M. Gür, I. D. Raistrick, and R. A. Huggins, J. Electrochem. Soc. 127, 2620 (1980).

${ }^{14}$ T. M. Gür, Ceram. Trans. 92, 3 (1999).

${ }^{15}$ A. Winkler, X. Guo, H. R. Siddiqui, P. L. Hagans, and J. T. Yates, Surf. Sci. 201, 419 (1988).

${ }^{16}$ N. Freyer, M. Kiskinova, G. Pirug, and H. P. Bonzel, Surf. Sci. 166, 206 (1986).

${ }^{17}$ J. L. Gland, B. Sexton, and G. B. Fisher, Surf. Sci. 95, 587 (1980).

${ }^{18}$ E. S. Putna, J. M. Vohs, and R. J. Gorte, Surf. Sci. 391, L1178 (1997). 